\title{
dRail: A Novel Physical Layout Methodology for Power Gated Circuits
}

\author{
Jatin N. Mistry ${ }^{1}$, John Biggs ${ }^{2}$, James Myers ${ }^{2}$, Bashir M. Al-Hashimi ${ }^{1}$, and \\ David Flynn ${ }^{2}$ \\ 1 School of Electronics \& Computer Science, University of Southampton, U.K. \\ \{jnm106, bmah\}@ecs.soton.ac.uk \\ 2 ARM Ltd., Cambridge, U.K. \{John.Biggs, James.Myers, David.Flynn\}@arm.com
}

\begin{abstract}
In this paper we present a physical layout methodology, called dRail, to allow power gated and non-power gated cells to be placed next to each other. This is unlike traditional voltage area layout which separates cells to prevent shorting of power supplies leading to impact on area, routing and power. To implement dRail, a modified standard cell architecture and physical layout is proposed. The methodology is validated by implementing power gating on the data engine in an $\mathrm{ARM}^{\circledR}$ Cortex $^{\mathrm{TM}}$ - A5 processor using a $65 \mathrm{~nm}$ library, and shows up to $38 \%$ reduction in area cost when compared to traditional voltage area layout.
\end{abstract}

Keywords: Physical Layout, Power Gating, Leakage

\section{Introduction}

Leakage power can be as dominant as dynamic power below $65 \mathrm{~nm}$ and poses a large source of power consumption in digital circuits [1]. A number of solutions have been proposed for reducing the leakage power dissipation of digital circuits which include the use of dual-threshold logic [2], application of reverse body bias [3] and power gating [4]. Power gating is proven to be the most effective and practical technique for reducing leakage power when logic is idle. For example, leakage power is lowered by $25 \mathrm{x}$ in the ARM926EJ-S ${ }^{\mathrm{TM}}$ with the application of power gating [5]. In this technique, the parts of a digital circuit which are to be powered down are connected to the VDD power supply through a high threshold voltage $\left(V_{t h}\right)$ PMOS power gating transistor, which creates a pseudo supply, often referred to as a virtual VDD (VVDD), on the drain side of the power gating transistor. When the PMOS transistor is disabled, this virtual supply is disconnected from the true supply eliminating leakage currents in the power gated logic [5].

To facilitate the implementation of power gating in an ASIC, the logic to be power gated is grouped into a voltage area in the physical layout [5]. This is due to the inherent abutment that occurs between the power and ground connections of adjacently placed cells in a traditional standard cell library, which would otherwise cause the switched VVDD to be shorted with the always on VDD in a power gating design. However, this physical separation has an area 
and routing cost on the design for a given performance target, as additional buffers and/or higher drive strength logic gates are inserted by the EDA tool to maintain performance [6] which also increases active power and will be shown in Section 3. Previous work has proposed to reduce the effects associated with the requirement for a voltage area by using distributed power gated rows $[7,8]$. The use of a custom standard cell library has also been proposed which allows two power supplies to be routed through each gate and duplicate gates are created for connecting to either of the supplies [9].

In this paper we propose a new physical layout design methodology, called dRail, which allows both power gated and non-power gated cells to be placed next to each other. This is unlike traditional voltage area layout [5] which separates power gated logic to prevent shorting of the switched and un-switched supplies. To achieve the dRail physical layout, first the standard cells are altered to stop them sharing the same power and ground supplies and prevents shorting of the switched and un-switched supplies without introducing additional cost to the standard cell architecture. Secondly, a modified cell layout is proposed to allow multiple supplies to be routed to every cell in the layout. The rest of this paper is organised as follows. Section 2 first describes the limitations of traditional voltage area layout before explaining the modified standard cell architecture and layout of the proposed dRail technique. Section 3 presents the validation of the proposed dRail methodology by implementation on a Cortex-A5. Section 4 concludes the paper.

\section{Proposed Technique}

The proposed dRail methodology allows both power gated and non-power gated cells to be placed next to each other to alleviate the need for a voltage area used in traditional power gating layout. dRail is achieved in two parts: firstly, the standard cell architecture is modified such that adjacent standard cells do not share the same power and ground by cutting back the power and ground connections. Secondly, the layout is modified to introduce a routing channel between site rows to allow both an always-on and switched supply to be routed to every cell.

Before the proposed dRail methodology is introduced, we first explain the limitations of the traditional voltage area layout used for power gated designs. Current standard cell gate libraries are designed such that the power (VDD) and ground (VSS) connections, usually in Metal1 (M1) (assumed for the rest of this paper), abut with adjacent cells when placed in a standard cell site row, Fig. 1. To ensure an uninterrupted VDD/VSS connection is available across the entire site row, any empty space is filled with M1 to create continuous M1 connections across the top and bottom of the site row which are referred to as rails. To prevent the always on VDD and switched VVDD supplies being shorted in the physical layout of a traditional power gated design, a voltage area is created [5] to separate the power gated logic from the always on logic denoted by the dashed line in Fig. 1. It should be noted that in this paper we assume a shared 


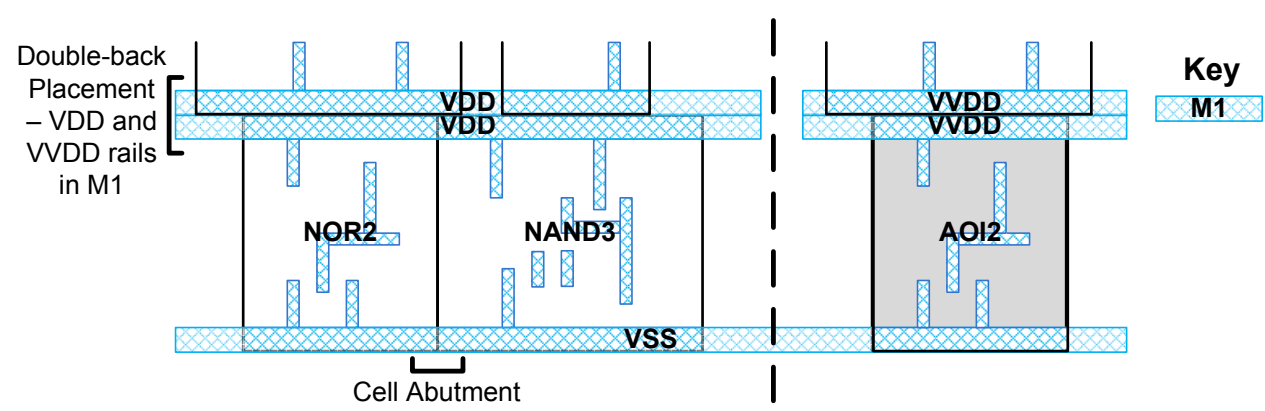

Fig. 1. Layout of power gating with traditional standard cell library and voltage area [5]. Note: break in the power supply rail and separation of shaded power gated cell

$\mathrm{N}$ and $\mathrm{P}$ well across voltage areas and a single switched VDD supply rail, as shown in Fig. 1, however a switched VSS supply rail is equally applicable. This separation can cause a greater distance between logically connected cells to arise which requires the addition of extra gates to maintain performance, resulting in area, routing and power overhead [6] (Section 3).

\subsection{Modified Standard Cell Architecture}

To overcome the requirement for a voltage area, and allow gates connecting to different power supplies to be placed adjacently, we propose to break, or 'de-rail', the continuous M1 rail across the standard cells to stop cells sharing the same power supplies, as in Fig. 1. To achieve this, we propose to shrink the power and ground (PG) pins of the standard cells so they no longer abut as shown in Fig. 2. Both the VDD and VSS connection are shrunk to allow the dRail technique to be used for switched VDD and/or VSS. Breaking the continuous M1 rail across the top and bottom of the site row means that each standard cell now has an independent VDD and VSS pin which can be connected to the necessary power supply, and will be shown in Section 2.2. The power gates are also modified as shown to enable them to be placed amongst the standard cells. To ensure the alterations shown in Fig. 2 do not introduce M1 spacing violations, the PG pins are cropped by $\frac{1}{2}$ the M1 design rule spacing from the edge. An added advantage of the proposed standard cell architecture is its versatility. The bounding box of the standard cell remains unchanged and therefore the cell occupies exactly the same area in placement. As the PG connections are only shrunk and the underlying function of the standard cell is unchanged, the cells can be used in a traditional placement flow by routing continuous M1 rails across the top and bottom of the cell rows with no change in power, performance or area.

\section{2 dRail Layout}

To demonstrate how the proposed modified standard cells, Section 2.1, can be used for a dRail layout, we convert the example shown in Fig. 1 with a single switched VVDD supply into a dRail layout. The layout is shown in Fig. 3 and 


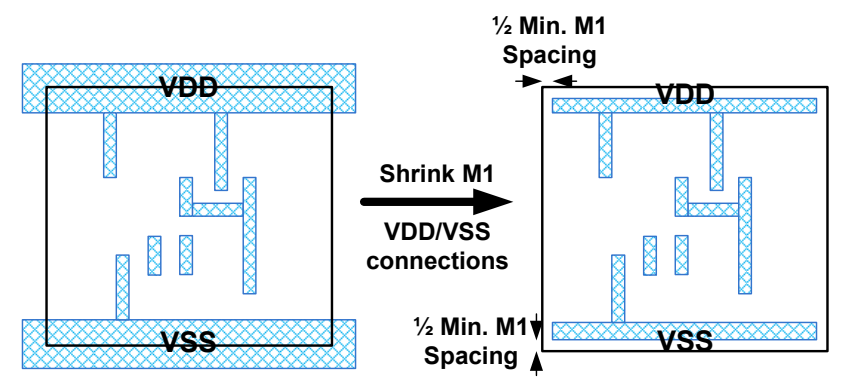

Fig. 2. Shrinking of VDD and VSS pins to stop power and ground abutment

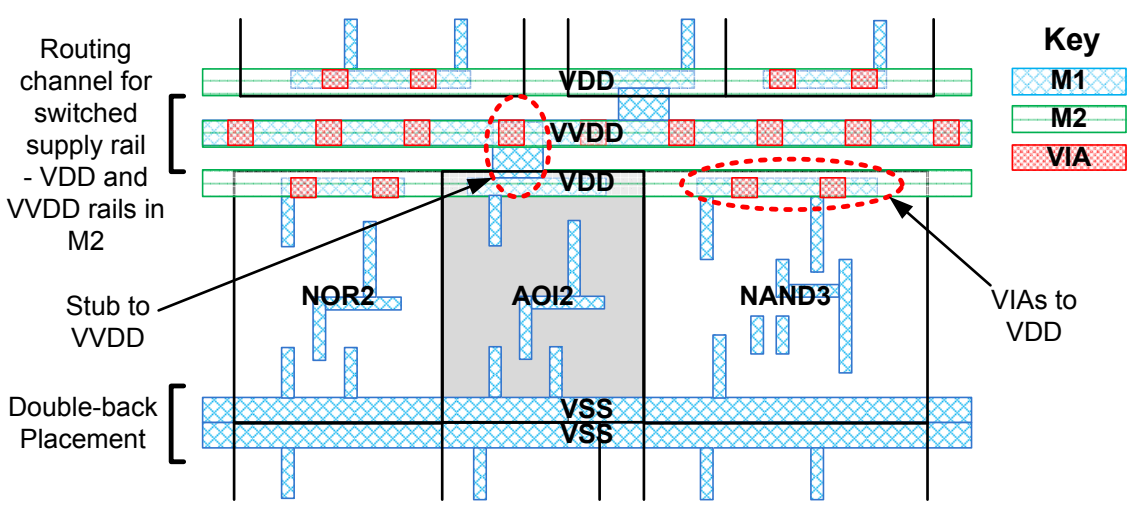

Fig. 3. Proposed dRail layout with a single switched supply rail, VVDD

there are three key features. Firstly, unlike traditional voltage area layout where M1 is used to create a continuous VDD or VVDD rail across the top of the site row, Fig. 1, Metal2 (M2) is used to create a continuous VDD rail. This means that only cells that need to connect to this supply rail can be connected with a VIA between the rail and the VDD pin as demonstrated on the NOR2 and NAND3 gates in Fig. 3. Secondly, instead of traditional double-back placement as was shown in Fig. 1, a small routing channel is introduced between the site rows to accommodate the switched VVDD supply rail which is routed on both M1 and M2. This allows the AOI2 cell that had to be separated into a voltage area in Fig. 1, to now be placed adjacent to the always on cells and is connected to the VVDD with an M1 stub as shown in Fig. 3. It should be noted that in the implementation of dRail the $\mathrm{N}$ well is common to both the always-on and power gated cells which means the power gated cells are reverse body biased when they are shut down. Thirdly, in this example, the VSS supply is unswitched, so the rows are placed double-back for VSS and a continuous M1 rail is created to ensure an uninterrupted connection along the site row. The example given here is for a single switched rail, however, a switched VVDD and VVSS rail, such as is found in Zig-Zag power gating [8], can also be achieved with the same M2 and routing channel layout employed on both sides of the site row.

To achieve this layout small modifications are required to a power gating physical design flow using standard EDA tools. We assume the use of the 


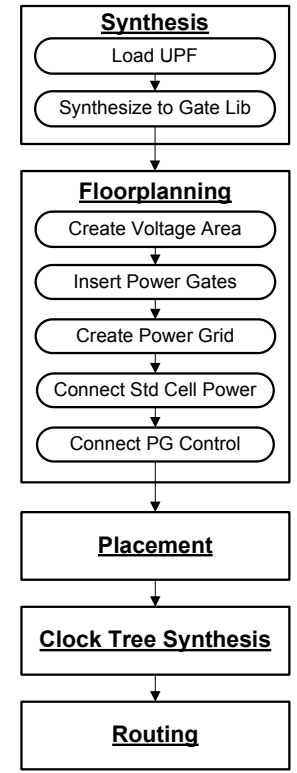

(a)

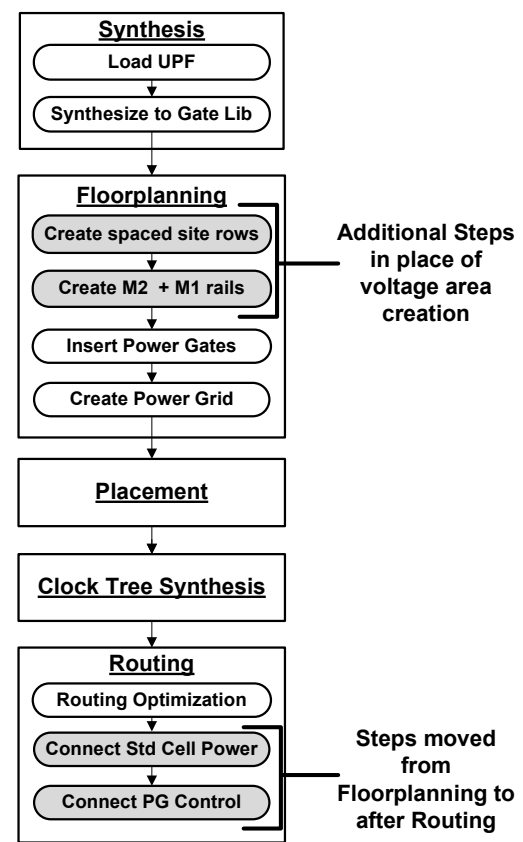

(b)

Fig. 4. Power gating physical design flow for (a) traditional voltage area (b) dRail

IEEE1801 UPF standard, a leading power design intent standard for defining the strategy of a multi-voltage or power gated design [10]. The physical design flow of a power gated circuit using dRail is shown in Fig. 4(b) and shows some subtle differences to a traditional power gated design flow using a voltage area, Fig. 4(a), which are highlighted. The synthesis stage is unchanged, however, it must be noted that the UPF file used in the dRail physical design flow must define the power gates in the 'DEFAULT' global voltage area and not within power domains as would traditionally be done. This ensures the EDA tools do not expect the power gates to be placed inside a voltage area, which in a dRail layout do not exist. There are a number of changes in floorplanning with the most important exclusion being the creation of a voltage area. Instead, the site rows must be carefully positioned to create the routing channel seen in Fig. 3 and the M1 and M2 rails must be routed in the correct locations. Furthermore, since no voltage area is used in dRail, it is recommended that the power gates are placed in a grid pattern throughout the dRail physical layout as opposed to rings which can be used in a voltage area layout. These steps can be automated in the implementation scripts. Placement, clock tree synthesis and routing remain unchanged, but the connection of the standard cells to the power rails is postponed until after routing. This is because the location of the standard cells are not fixed until this point and the stub and VIA connections required to connect the power to the modified standard cells (Fig. 3) would be incorrect had they been done earlier. 


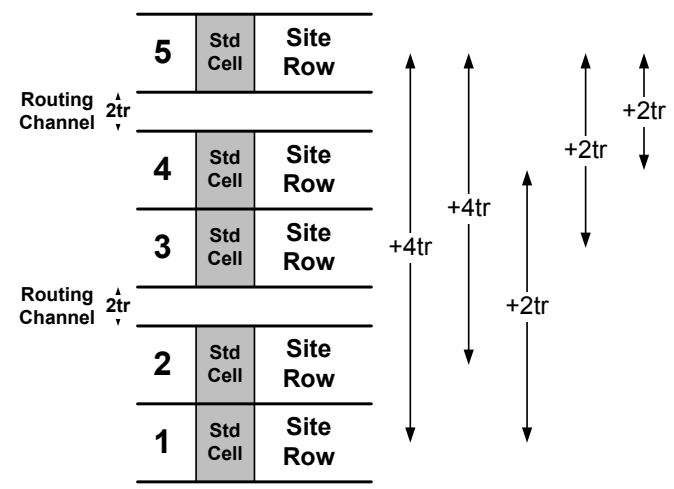

Fig. 5. Area overhead between standard cells (spreading)in dRail. $\operatorname{tr}=1$ Routing track

\section{3 dRail Overheads}

The proposed dRail design methodology introduces three overheads that must be considered in the physical layout. Firstly, the extra power routing done on M2 in the dRail layout, Fig. 3, creates routing blockage which can offset the routing improvements achievable with the dRail layout. Secondly, the additional routing channel between the site rows shown in Fig. 3 for inclusion of the switched supply rail results in 'dead' space as it cannot be used for placement. The area taken up by this additional routing space is the equivalent to one routing track per switched rail, per site row and is therefore dependent on the gate library being used. As an example, with a 12 track gate library i.e. each standard cell is 12 routing tracks in height, for a given number of site rows $x$, the loss of placement area for one additional power supply rail in dRail is $\frac{x}{12}$. Finally, the routing channel introduced between the site rows also results in spreading of the standard cells. For example, in the case with one switched rail, two standard cells placed directly opposite each other 3 site rows apart - e.g. rows 1 and 4 or 2 and 5 in Fig. 5 - results in the distance between them increasing by 2-4 routing tracks which can require the insertion of additional buffers to maintain performance. These overheads have an impact on the overall physical layout when using dRail, however bounded use of the dRail physical layout can minimise these overheads and improve the overall area and routing cost in a power gating physical layout and will be shown in Section 3 .

\section{Experimental Results}

Three experiments were carried out to investigate the proposed dRail methodology. The first shows the impact of the overheads in the dRail layout methodology described in Section 2.3. The second and third show how the dRail layout can be bounded to reduce the effect of the overheads and hence improve the area cost associated with traditional voltage area layout. The experiments were carried out by power gating the data engine (DE) (floating point unit plus $\mathrm{NEON}^{\mathrm{TM}}$ unit) 


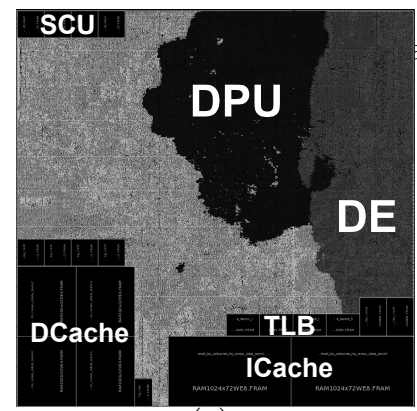

(a)

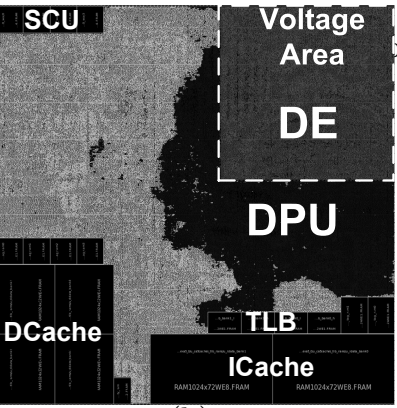

(b)

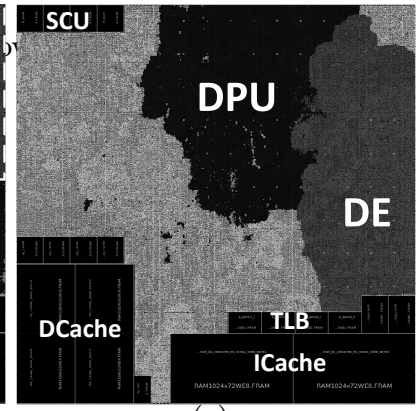

(c)

Fig. 6. Floorplan of A5 with interaction of Data Engine and Data Processing Unit (a) no power gating (b) DE power gated with voltage area (c) DE power gated with dRail

Table 1. Area, routing and power in no power gating and power gating with voltage area [5], and proposed dRail with difference to no power gating shown

\begin{tabular}{|l|c|c|c|c|c|}
\hline & No Power Gating & Voltage Area [5] & Diff (\%) & Proposed dRail & Diff (\%) \\
\hline $\begin{array}{l}\text { Total Cell Area } \\
\left(\mu m^{2}\right)\end{array}$ & $1,246,592$ & $1,286,710$ & 3.2 & $1,258,415$ & 0.9 \\
\hline $\begin{array}{l}\text { of which: DE } \\
\text { Area }\left(\mu m^{2}\right)\end{array}$ & 206,007 & 216,407 & 5 & 211,277 & 2.6 \\
\hline $\begin{array}{l}\text { PG Area Cost } \\
\left(\mu m^{2}\right)\end{array}$ & 0 & 2180 & - & 85,326 & - \\
\hline $\begin{array}{l}\text { Total Placement } \\
\text { Area }\left(\mu m^{2}\right)\end{array}$ & $1,246,592$ & $1,288,890$ & 3.4 & $1,348,422$ & 8.2 \\
\hline $\begin{array}{l}\text { Routing Length } \\
(\mu m)\end{array}$ & $6,819,157$ & $7,329,862$ & 7.5 & $6,783,361$ & -0.5 \\
\hline $\begin{array}{l}\text { Normalised } \\
\text { Active Power }\end{array}$ & 1 & 1.08 & - & 1.01 & - \\
\hline
\end{tabular}

in an ARM Cortex-A5 processor as its close interaction and tightly coupled nature with the rest of the data processing unit (DPU) makes it difficult to power gate. The processor was synthesized using a TSMC 65LP ARM Artisan ${ }^{\circledR}$ library modified for use with dRail and consisted of a single core, 16k Level-1 data and instruction cache, TLB cache and snoop control unit cache (SCU). All implementations targeted and achieved the same clock frequency and were fully place and routed using a UPF driven power gating flow with the Synopsys EDA tools. To ensure comparison of results was fair, the placement of the caches and silicon core area $(1245 \mu \mathrm{m} \times 1244.2 \mu \mathrm{m})$ was kept fixed in all implementations.

\subsection{Effect of dRail Overheads}

An implementation of the Cortex-A5 was created without power gating and served as the baseline area, routing length and active power for the power gating implementations. Its floorplan can be seen in Fig. 6(a), and in particular, notice how the DE and DPU closely interact. Conversely, a floorplan of the same Cortex-A5 with a traditional voltage area power gating layout [5] used for the DE can be seen in Fig. 6(b) with the voltage area in the top right corner. As can be seen, the DPU is 'pulled' towards the DE and is done to reduce the distance between logically connected gates and maintain performance, but the voltage area shows a clear boundary (or guard band) between the two which results in $2180 \mu m^{2}$ of 'dead' area which we refer to as power gating area cost in Table 1. 
The separation of these gates consequently has a $3.2 \%$ cost in total cell area from the addition of extra and larger gates and can be seen in Table 1 . When coupled with the power gating area cost, the voltage area layout results in in a $3.4 \%$ increase in total placement area with respect to no power gating, and a $7.5 \%$ increase in routing. The increase in cell area and routing length consequently have an impact on the active power of the design which increases by $8 \%$.

Fig. 6(c) shows the floorplan of the Cortex-A5 when using dRail throughout the entire physical layout. Unlike traditional voltage area layout, using dRail gives the EDA tool the freedom to place the standard cells anywhere resulting in a similar tightly coupled layout as the design without power gating, Fig. 6(a). The increase in total and DE cell areas are subsequently lower when compared to using a voltage area layout (Table 1) but is still higher than no power gating because of the spreading that occurs in dRail and hence the addition of extra and larger gates. Interestingly, routing length is reduced even when compared to no power gating and can be explained by a reduction in routing congestion from the introduction of the routing channels. Furthermore, the reductions in cell area and routing results in active power becoming comparable to no power gating. However, the overheads discussed in Section 2.3 result in the blanket use of dRail amounting to poor total placement area results in this test case. This is because the placement area wasted from the inclusion of routing channels incurs a large power gating area cost of $85,326 \mu \mathrm{m}^{2}$. This results in a higher total placement area than the voltage area layout and shows how no consideration of the impact of the overheads can result in an overall negative effect in terms of placement area.

\subsection{Bounded dRail}

To reduce the dRail overheads, the versatility of the proposed dRail standard cell architecture can be exploited to create bounded dRail layouts rather than using it throughout the entire physical layout. An example of this is shown in Fig. 7(a) where the right of the floorplan has a dRail layout with VDD and VVDD available for placement of power gated and always on cells together, and on the left of the floorplan, a traditional placement is used with only VDD available to eliminate the dRail spreading area cost in this placement area. As can be seen, the DE is entirely enclosed in the dRail boundary but the availability of the VDD supply rail allows logic gates from the DPU to be 'pulled' into the boundary to reduce the distance between logically connected gates. This is unlike a voltage area layout where the boundary enforced is exclusive to only the DE cells, Fig. 6 (b), and shows the strength of the proposed dRail layout methodology. Table 2 shows the results achieved with this bounded 'Partial dRail' implementation. As can be seen, bounded dRail improves upon the increase in total and DE cell area as well as routing length and power when compared to a voltage area layout but is also better than the blanket use of dRail throughout the layout (Table 1) because of a reduced impact from standard cell spreading overheads. The bounded use of dRail in this design has also helped to improve the power gating area cost compared to a blanket dRail implementation (Table 1). This 


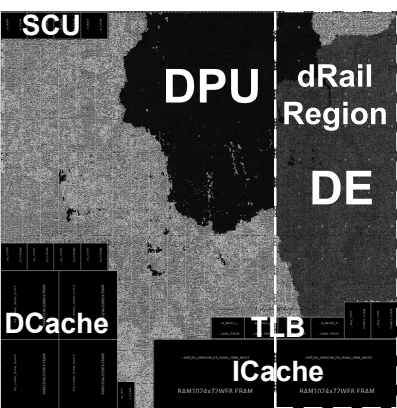

(a)

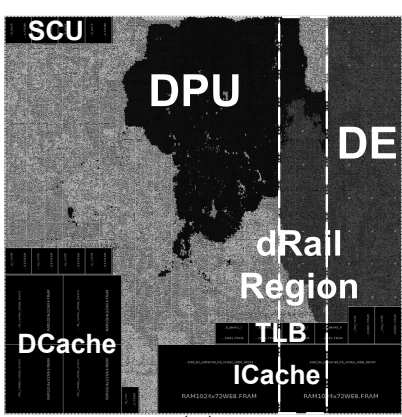

(b)

Fig. 7. Floorplan of A5 with interaction of Data Engine and Data Processing Unit (a) DE power gated with partial dRail (b) DE power gated with dRail on interface

Table 2. Area, routing and power in no power gating and power gating with voltage area [5], partial dRail, and interface dRail with difference to no power gating shown

\begin{tabular}{|l|c|c|c|c|c|c|c|}
\hline & $\begin{array}{c}\text { No Power } \\
\text { Gating }\end{array}$ & $\begin{array}{c}\text { Voltage } \\
\text { Area [5] }\end{array}$ & $\begin{array}{c}\text { Diff } \\
(\%)\end{array}$ & $\begin{array}{c}\text { Proposed } \\
\text { Partial dRail }\end{array}$ & $\begin{array}{c}\text { Diff } \\
(\%)\end{array}$ & $\begin{array}{c}\text { Proposed } \\
\text { Interface dRail }\end{array}$ & $\begin{array}{l}\text { Diff } \\
(\%)\end{array}$ \\
\hline $\begin{array}{l}\text { Total Cell Area } \\
\left(\mu \mathrm{m}^{2}\right)\end{array}$ & $1,246,592$ & $1,286,710$ & 3.2 & $1,236,267$ & -0.8 & $1,236,528$ & -0.8 \\
\hline $\begin{array}{l}\text { of which: DE } \\
\text { Area }\left(\mu \mathrm{m}^{2}\right)\end{array}$ & 206,007 & 216,407 & 5 & 203,735 & -1.1 & 203,587 & -1.2 \\
\hline $\begin{array}{l}\text { PG Area Cost } \\
\left(\mu m^{2}\right)\end{array}$ & 0 & 2180 & - & 35,752 & - & 18,359 & - \\
\hline $\begin{array}{l}\text { Total Placement } \\
\text { Area }\left(\mu m^{2}\right)\end{array}$ & $1,246,592$ & $1,288,890$ & 3.4 & $1,294,167$ & 3.8 & $1,278,623$ & 2.6 \\
\hline $\begin{array}{l}\text { Routing Length } \\
(\mu m)\end{array}$ & $6,819,157$ & $7,329,862$ & 7.5 & $6,574,952$ & -3.6 & $6,506,849$ & -4.6 \\
\hline $\begin{array}{l}\text { Normalised } \\
\text { Active Power }\end{array}$ & 1 & 1.08 & - & 0.99 & - & 0.99 & - \\
\hline
\end{tabular}

brings the total placement area down to a comparable value to the voltage area layout whilst eliminating the $8 \%$ increase in active power.

An interesting thing to observe in Fig. 7(a) is that the interaction of the DPU and DE is largely isolated to the boundary. For this reason a second bounded implementation was created, Fig. 7(b), where dRail is only used on the interface of the two blocks to further minimise area overhead incurred in the DE region. The far right of the floorplan uses traditional placement with only VVDD for DE standard cells, and the left of the floorplan has only VDD for always-on standard cells. The results from this 'Interface dRail' physical layout are shown in Table 2. As can be seen, the area, routing and power are very similar to the 'Partial dRail' implementation but the power gating area cost has been reduced further. In this case an improvement of $38 \%$ is achieved over the voltage area layout when comparing the total placement area, whilst simultaneously eliminating the $8 \%$ increase in active power. These bounded dRail implementations demonstrate the versatility of the proposed methodology and shows how many power domains could be interleaved using the 'interface dRail' approach. Similarly, although one switched power rail and one power domain is shown in this test case, dRail with bounded placement has the potential for multiple switched rails for multiple power domains such as Zig-Zag power gating [8] or SoC interconnect. 


\section{Conclusion}

This paper has proposed a new physical layout methodology, called dRail, for reducing the area, routing and power cost associated with using a voltage area in power gated designs by enabling power gated and non-power gated cells to be placed adjacent to one another. This is unlike traditional power gating layout where the standard cells are separated into a voltage area to prevent shorting of the switched and un-switched supplies. Experimental results on an ARM CortexA5 showed that bounded use of dRail can provide the largest improvements in area, routing and power whilst meeting the same performance target. The dRail methodology proposed in this paper is targeted at power gating in designs with highly interleaving logic such as zig-zag power gating or power gating in SoC fabric and builds on the multi-voltage EDA tools and flows with it being fully compatible with standard UPF power intent. dRail also has the potential for use in multi-VDD layout, but requires careful consideration of the back/forward biasing that could occur.

\section{References}

1. Agarwal A., Mukhopadhyay S., Raychowdhury A., Roy K., Kim C.H.: Leakage Power Analysis and Reduction for Nanoscale Circuits. IEEE Micro. vol. 26, pp. 68-80. IEEE (2006)

2. Wei L., Chen Z., Roy K., Johnson M.C., Ye Y., De V.K.: Design and Optimization of Dual Threshold Circuits for Low-Voltage Low Power Applications. IEEE Transactions on Very Large Scale Integration (VLSI) Systems. vol. 7, pp. 16-24. IEEE (1999)

3. Tschanz J.W., Narendra S.G., Ye Y., Bloechel B.A., Borkar S., De V.: Dynamic Sleep Transistor and Body Bias for Active Leakage Power Control of Microprocessors. IEEE Journal of Solid-State Circuits. vol. 38, pp. 1838-1845. IEEE (2000)

4. Mutoh S., Douseki T., Matsuya Y., Aoko T., Shigematsu S., Yamada J.: 1-V Power Supply High-Speed Digital Circuit Technology with Multithreshold-Voltage CMOS. IEEE Journal of Solid-State Circuits. vol. 30, pp. 847-854. IEEE (1995)

5. Keating M., Flynn D., Aitken R., Gibbons A., Shi K.: Low Power Methodology Manual. Springer (2007)

6. Weste N.H., Harris D. M.: CMOS VLSI Design: A Circuits and Systems Perspective. 4th Ed. Addison-Wesley (2011)

7. Sathanur A., Benini L., Macii A., Macii E., Poncino M.: Row-Based Power-Gating: A Novel Sleep Transistor Insertion Methodology for Leakage Power Optimization in Nanometer CMOS Circuits. IEEE Transactions on Very Large Scale Integration (VLSI) Systems. vol 19, pp. 469-482. IEEE (2011)

8. Shin Y., Paik S., Kim H.,: Semicustom Design of Zigzag Power-Gated Circuits in Standard Cell Elements. IEEE Transactions On Computer-Aided Design of Integrated Circuits and Systems. vol. 28, pp. 327-339. IEEE (2009)

9. Yeh C., Kang Y.,: Cell-Based Layout Techniques Supporting Gate-Level Voltage Scaling for Low Power. IEEE Transactions On Very Large Scale Integration (VLSI) Systems. vol. 9, pp. 983-986. IEEE (2001)

10. IEEE1801 Standard, http://standards.ieee.org/findstds/standard/ 1801-2009.html 